\title{
CARACTERÍSTICAS SOCIODEMOGRÁFICAS DE IDOSOS COM REDUÇÃO DA ATIVIDADE FÍ́SICA ACOMPANHADOS NA ATENÇÃO PRIMÁRIA À SAÚDE
}

Patrícia Rosa Gonçalves Leta; Universidade Federal do Paraná; patriciarg|@hotmail.com; Márcia Marrocos Aristides Barbiero; Universidade Federal do Paraná; marciamarrocos@gmail.com;

Reuber Lima de Souza; Universidade Federal do Paraná; reuber_souza@hotmail.com; Fatima Denise Padilha Baran; Universidade Federal do Paraná; fatima_enfermagem@yahoo.com.br Aline de Sousa Falcão; Universidade Federal do Paraná; alinesousafalcão@hotmail.com; Kamila Alves Brasileiro; Universidade Federal do Paraná; kamilabrasileiro@ufpr.br Susanne Elero Betiolli; Universidade Federal do Paraná; susanne.elero@yahoo.com.br Maria Helena Lenardt; Universidade Federal do Paraná; curitiba.helena@gmail.com

\section{RESUMO}

Introdução: O conhecimento das características sociodemográficas permite, ao profissional de saúde, a elaboração de estratégias mais eficazes voltadas às necessidades e realidade dos idosos. Objetivo: Caracterizar o perfil sociodemográfico de idosos com redução da atividade física da atenção primária à saúde. Métodos: trata-se de estudo quantitativo de corte transversal, realizado em uma Unidade Básica de Saúde de Curitiba-Paraná (Brasil), com amostra constituída por 389 idosos ( $\geq 60$ anos). Realizou-se rastreio cognitivo, na sequência foi aplicado questionário Sociodemográfico, e realizada avaliação do nível de atividade física, mediante o Minnesota Leisure Time Activities Questionnaire. Realizaram-se análises estatísticas descritivas. O projeto foi aprovado pelo Comitê de Ética em Pesquisa em Seres Humanos sob número CEP/SD 2918847. Resultados: dos 389 idosos, 81 (20,8\%) apresentaram redução de atividade física e, destes, 55 (67,9\%) eram mulheres, $53(65,4 \%)$ de raça branca, $36(44,4 \%)$ com idade de 60 a 69 anos, 39 (48,1\%) católicos, 36 $(44,4 \%)$ casados, $19(23,45 \%)$ residem sozinhos, $43(53,1 \%)$ são aposentados e $49(60,5 \%)$ recebem até 2 salários mínimos. Conclusão: As características sociodemográficas dos idosos com redução da atividade física apontam para o sexo feminino, aposentados e de baixa renda. Esses resultados indicam a necessidade de se considerarem estratégias efetivas e de baixo custo a serem implementadas na atenção primária à saúde, e incorporada aos cuidados gerontológicos, que visem o incentivo à prática da atividade física, considerando os benefícios à saúde dos idosos.

Palavras-chave: Idoso; Atenção Primária à Saúde; Exercício Físico.

Agradecimentos: À Coordenação de Aperfeiçoamento de Pessoal de Nível Superior (CAPES), pela concessão de bolsa de mestrado pelo Programa de Pós-Graduação em Enfermagem/UFPR. 